persive delay; indeed, the wind density implied is lower than that expected for an isolated B star. The spectral type and photometry indicate that the companion mass is greater than 10 solar masses, and optical spectroscopy may allow a test of this conclusion.

These two companions are the most massive objects found orbiting radio pulsars. Also announced in Aspen (A. Wolszczan, Pennsylvania State University) was the lightest: a Moon-sized body in a 25-day orbit around the pulsar $\mathrm{B} 1257+12$, a pulsar that had previously been deduced to have two planets a few times bigger than the Earth ${ }^{6}$. Wolszczan also laid to rest any serious doubt that the original double-oscillatory period variations observed in this pulsar are gravitationally induced by orbiting planets, by announcing the detection of the predicted secular variations of the bodies' orbital parameters due to their mutual perturbations ${ }^{7}$.

Another intriguing candidate for a pulsar-planetary system came in the form of quasi-oscillatory phase variations of the slow pulsar B1828-11 (Lyne), which might be consistent with free precession of the neutron $\operatorname{star}^{8}$, but which can also be decomposed into the sum of three keplerian orbits.

Indirect evidence for a planet orbiting the binary pulsar B1620-26, in the globular cluster M4, came last year from an anomalously large second-derivative of the period ${ }^{9,10}$. Measurements of a period third-derivative (D. C. Backer, University of California, Berkeley, and myself) give a characteristic orbital timescale (for a circular orbit) of 160 years, and an implied mass of 80 times that of the Earth. However, when eccentric orbits are considered (F. C. Michel, Rice University; S. Sigurdsson, University of California, San Diego) the allowed companion mass rises to a solar mass or more. Consideration of dynamical instability and secular perturbation of the orbit of the inner body by that of the outer leads most naturally to a stellar-mass companion, with an orbital period measured in centuries (F. A. Rasio, Institute for Advanced Studies, Princeton). Such triple systems are naturally expected in the dense cores of globu-

1. Taylor, J. H., Manchester, R. N. \& Lyne, A. G. Astrophys. J. suppl.Ser. 88.529-568(1993)

2. Backer, D. C., Kulkarni, S. R., Heiles, C., Davis, M. M. \& Goss, W. M. Nature 300, 615-618(1982).

3. Kulkarni, S. R. \& Narayan, R. Astrophys. J. 335, 755-768 (1988).

4. Narayan, R., Fruchter, A. S., Kulkarni, S. R. \& Romani R. W. in Accretion Powered Compact Binaries (ed. Mauche C. W.) 451-456 (Cambridge Univ. Press, 1990)

5. Johnston, S. et ai. Astrophys. J. 387, L37-L41 (1992).

6. Wolszczan, A. \& Frail, D. A. Nature 355, 145 (1992).

7. Malhotra. R. Astrophys. J. 407, 266-275 (1993).

8. Cordes, J. M. Planets around Pulsars (eds Phillips, J. A.

Thorsett, S. E. \& Kulkarni, S. R.) 43-60 (Astr. Soc. Pac. Conf. Ser. Vol. 36, 1993)

9. Backer, D. C., Foster, R. S. \& Sallmen. S. Nature 365 $817-819$ (1993)

10. Thorsett, S. E.. Arzoumanian, Z. \& Taylor, J. H. Astrophys. J. 412. L33- L36 (1993)

\section{A new angle on Mars}

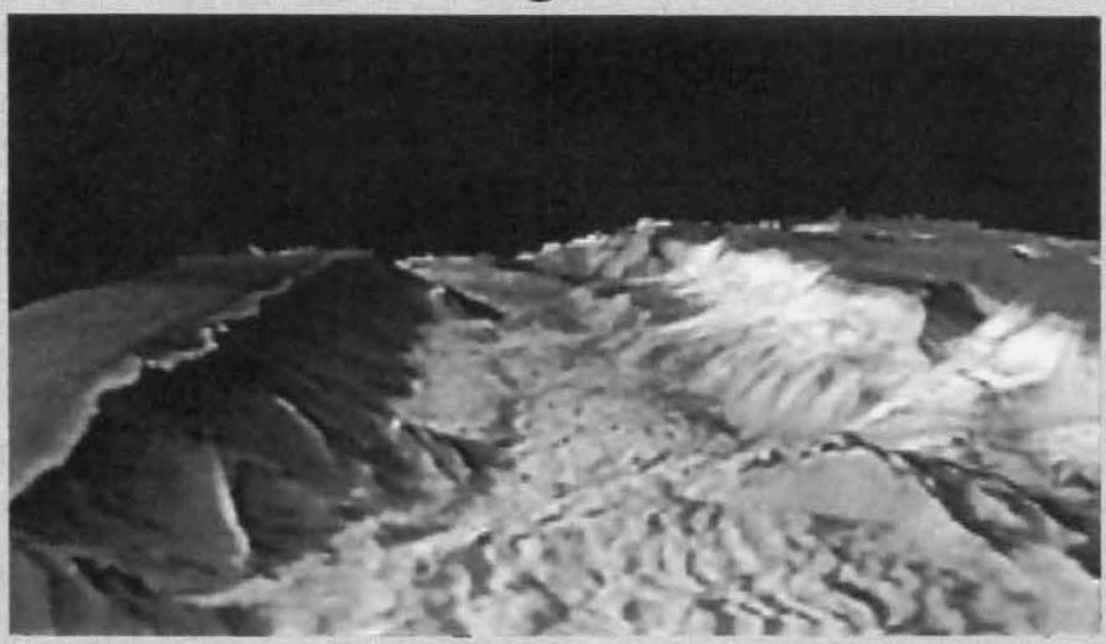

LOOKING at this image of the surface of Mars, one has the eerie impression of seeing the landscape from the window of an aeroplane. How is it possible to survey the planet this way?

The answer lies in stereo-pair images taken from the Viking Orbiter satellite and processed by G. Thornhill and co-workers (J. geophys. Res. 98, 2358123587; 1993). Software developed at University College London automatically matches several thousand points, making light work of what would pre. viously have been months of tedious manual labour. As the images are taken with different geometries, the two different relative positions of each point can be used to derive its height provided one knows the spacecraft's elevation and the camera direction.

The result is a grid of known heights (the obligatory acronym is a DEM, or digital elevation model). One image from the stereo pair is combined with this grid on the computer - essentially, the original photograph provides a pattern of light and shade to be draped

lar clusters, but the expected lifetime is short compared with that of the millisecond pulsar.

Caution is needed, though, in interpreting such timing irregularities. Systematic deviations from a simple spin-down model have also now been observed, albeit at a much lower level, in eight years of observations of the original millisecond pulsar B1937+21 (Kaspi). Because similar instabilities are not seen in comparable data for pulsar B1855+09 (an older pulsar, but discovered more recently), and multi-frequency observations seem to rule out propagation effects as the cause, the simplest conclusion is that intrinsic rotational instabilities are being observed. Although 'timing noise' is familiar in young, slow pulsars, this is the first time it has been observed in a recycled pulsar. over a scaffolding of heights.

A perspective view can be generated from any apparent height and direction above the surface. This particular im. age is the view along Tithonium Chasma, part of the great Valles Marineris chasm system, as it would appear from 30 kilometres up. The chasm is about 7 kilometres deep and 40 kilometres wide at this point, putting Colorado's Grand Canyon, a mere 1.9 kilometres deep, in the shade.

In the immediate foreground, part of the chasm wall has slumped in a landslide, spreading in folds and corrugations almost to the opposite wall of the channel. Those whose taste is more for huge impact craters or putative dried beds of ancient waterways will find them in the original paper.

Impressive as their images are, Thornhill and colleagues strike a mournful note in their introduction. If the Mars Observer were still with us, direct measurements of the martian topography from laser altimeter data could have been flooding in.

So a decade after its discovery, the natural limits of the precision of one of nature's most remarkable clocks are finally being explored; by coincidence, its stability, slightly better than one part in $10^{14}$ in a few years, is comparable to the best terrestrial atomic clocks. We now know that B1937+21 is young for a millisecond pulsar, however, and already observations of pulsar B1855+09 suggest it may reach stabilities of a few parts in $10^{15}$ or better. As such pulsars are discovered and studied, pulsar astronomers have good reason to expect that the next decade will prove as exciting as the last.

S. E. Thorsett is in the California Institute of Technology, Pasadena, California 91125, USA. 